\title{
Absorbing boundary conditions for wave-equation migration
}

\section{Robert W. Clayton* and Björn Engquist}

The standard boundary conditions used at the sides of a seismic section in wave-equation migration generate artificial reflections. These reflections from the edges of the computational grid appear as artifacts in the final section. Padding the section with zero traces on either side adds to the cost of migration and simply delays the inevitable reflections.

We develop stable absorbing boundary conditions that annihilate almost all of the artificial reflections. This is demonstrated analytically and with synthetic examples. The absorbing boundary conditions presented can be used with any of the different types of finite-difference wave-equation migration, at essentially no extra cost.

\section{INTRODUCTION}

One of the common procedures in processing seismic reflection data is migration. Migration places reflections from dipping beds in their correct locations and collapses diffractions. In wave-equation migration (Claerbout. 1970, 1976: Claerbout and Johnson, 1971), the recorded surface wave field is downward continued by solving a paraxial or one-way wave equation (backward in time). The actual downward continuation operator used in the computations is found by approximating the paraxial differential equations with finite differences. With this method, it is necessary to specify boundary conditions, and at the side boundaries of the section either zero-slope (Neumann) or zero-value (Dirichlet) end conditions are typically used (Claerbout, 1976). Although these boundary conditions are easy to implement, they have the unfortunate property of forcing the boundaries to act as perfect reflectors. Thus, reflections from dipping beds or diffractions that would be partially off the section after migration are reflected at the boundaries and appear as artifacts in the final section.

This problem is common to all calculations where artificial boundaries are introduced to limit the computational size. One scheme that is used to reduce the reflections is to pad the sides of the section with zero traces. This, however, adds to the cost of processing and does not completely remove the artifacts. To remove spurious reflections in a more direct and efficient manner, one would like to specify boundary conditions which absorb energy incident on the sides. This would add essentially no extra cost to the migration.

Absorbing boundary conditions for modeling schemes based on finite differences of the full acoustic or elastic wave equation have been found (Clayton and Engquist, 1977: Engquist and Majda, 1977: Reynolds, 1978). However, this problem differs from the one considered here in that we develop absorbing boundary conditions for paraxial equations. A somewhat different approach to the problem is used because of the different character of these equations. These boundary conditions substantially reduce spurious reflections compared to the ones currently used. Absorbing boundary conditions can be used in either the time or frequency domain, and for the various approximations of paraxial equations used in migration.

A brief review of paraxial equations is given first. The boundary conditions themselves are derived in the second section, which includes an example of their effective side reflection coefficients. The boundary conditions are illustrated with both a timeand a frequency-domain example. In the Appendix, explicit formulas are presented for the boundary

Manuscript received by the Editor Fobruary 1, 1979; revised manuscript received September 6. 1979.

* Department of Geophysics, Stanford University, Stanford, CA 94305.

$\doteqdot$ Department of Mathematics. University of California at Los Angeles, 405 Hilgard Ave.. Los Angeles. CA 90024.

0016-8033/80/0501-0895\$03.00 (C) 1980 Society of Exploration Geophysicists. All rights reserved. 


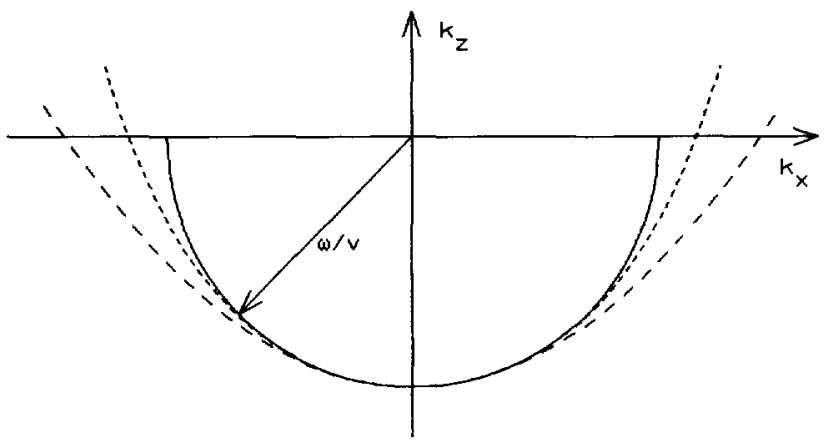

FIG. 1. Dispersion relations for paraxial wave equations. The semicircle is the dispersion relation of the exact paraxial wave equation. The other curves are the 15-degree (long dashed curve) and 45-degree approximations (short dashed curve) given by equations (4) and (5), respectively.

conditions, and a stability proof is given for a particular example.

\section{PARAXIAL WAVE EQUATIONS FOR MIGRATION}

Derivations of paraxial wave equations have been given by several authors (Claerbout, 1970, 1976; Clayton and Engquist, 1977); here we restrict ourselves to a brief review. The dispersion relation for the full acoustic wave equation

$$
P_{x x}+P_{z z}-\frac{1}{\imath^{2}} P_{t t}=0
$$

where $P$ is the wave field amplitude and $I$ ' is velocity, is the circle

$$
k_{x}^{2}+k_{z}^{2}-\frac{\omega^{2}}{v^{2}}=0
$$

where $k_{x}$ and $k_{z}$ are the horizontal and vertical wavenumbers, respectively, and $\omega$ is the temporal frequency.

The full wave equation supports waves moving in both downgoing $(+z)$ and upgoing $(-z)$ directions. The paraxial equation for upgoing waves uses the semicircle dispersion relation

$$
k_{z}=-\left(\frac{\omega^{2}}{v^{2}}-k_{x}^{2}\right)^{1 / 2} .
$$

To cast equation (3) as a differential equation, it is necessary to approximate the square root as a rational function of $\omega$ and $k_{x}$. Two approximations commonly known as the 15-degree and 45-degree approximations, respectively, are

$$
k_{z}=-\frac{\omega}{v}\left(1-\frac{1}{2} \frac{v^{2} k_{x}^{2}}{\omega^{2}}\right),
$$

and

$$
k_{z}=-\frac{\omega}{v} \frac{1-\frac{3}{4} \frac{v^{2} k_{x}^{2}}{\omega^{2}}}{1-\frac{1}{4} \frac{v^{2} k_{x}^{2}}{\omega^{2}}}
$$

Equation (4) is the result of a linear approximation of the square root, while equation (5) comes from a bilinear approximation. The dispersion relations given by equations (3), (4), and (5) are shown in Figure 1. Using the following sign convention for the Fourier transforms

$$
\begin{gathered}
P\left(k_{x}, k_{z}, \omega\right)=\iiint P(x, z, t) . \\
\cdot e^{i\left(k_{x}, x^{*}+k_{z} z^{z-\omega t)}\right.} d x d z d t,
\end{gathered}
$$

we find the differential equations corresponding to equations (4) and (5) are, respectively,

$$
P_{z t}+\frac{\nu}{2} P_{x \cdot x}-\frac{1}{\nu} P_{t t}=0,
$$

and

$$
P_{z t t}-\frac{v^{2}}{4} P_{x x z}+\frac{3 v^{\prime}}{4} P_{. r x t}-\frac{1}{v} P_{t t t}=0 .
$$

For computational purposes, it is often convenient to use a retarded time coordinate system

$$
t^{\prime}=t+\frac{z}{v}
$$

which has the effect of reducing the equations (for constant velocity) to

$$
P_{z t^{\prime}}+\frac{v}{2} P_{x x}=0
$$


and

$$
P_{z t^{\prime} t^{\prime}}-\frac{v^{2}}{4} P_{x \cdot x z}+\frac{v}{2} P_{x x t^{\prime}}=0
$$

A method for finding higher order paraxial equations is given in Clayton and Engquist (1977). The paraxial equations for downgoing waves can be found from equations (3) through (10) by replacing $k_{z}$ by $-k_{z}$, and $\partial / \partial_{z}$ by $-\partial / \partial_{z}$.

In the wave-equation migration process, paraxial equations such as (6) or (7) are solved together with the recorded data $P(x, z=0, t) \quad(A \leq x \leq B$, $0 \leq t \leq T)$ as initial conditions. $P$ is assumed to vanish for $t<0$ and $t>T$. The wave field $P(x, z, t)$ is calculated for $z>0$ by approximating the differential equations [for example, equations (9) or (10)] with difference approximations. The final migrated section is taken as the slice $P(x, z, t=0)$. For a more detailed description, see Clacrbout (1976). To perform these calculations, extra conditions at the artificial side boundaries $(x=A$ and $x=B)$ are needed on $P$, and that is where we want absorbing boundary conditions.

\section{ABSORBING BOUNDARY CONDITIONS}

To derive the absorbing boundary conditions, consider an upgoing monochromatic plane wave incident upon the right side boundary $\left(k_{x}, \omega<0\right)$ of the form

$$
P_{I}=e^{i\left(k_{x}, r-k_{z} z-\omega r\right)} .
$$

The side boundary will set up a reffected wave

$$
R P_{R}=R e^{i\left(-k_{x} x-k_{z} z-\omega t\right)},
$$

where $R$ is the effective reflection coefficient of the edge of the grid.

The boundary condition operator, which we denote as $B$, governs the total wave field at the edge in the following fashion

$$
B\left(P_{I}+R P_{R}\right)=0 .
$$

We restrict ourselves to linear boundary conditions so that the effective reflection coefficient is found to be

$$
R=-\frac{B\left(P_{I}\right)}{B\left(P_{R}\right)} .
$$

Since the wave fields themselves are functions of $k_{z}, k_{r}$, and $\omega$, and the relationship between $k_{z}$ and the other two variables is fixed by the paraxial equation used in the interior, we can write equation (14) as

$$
R=-\frac{B\left(k_{x}, \omega\right)}{B\left(-k_{x}, \omega\right)} .
$$

For absorbing boundary conditions, we wish to minimize $|R|$, keeping in mind that the boundary conditions should not introduce instabilities into the solution. Clearly, $|R|$ will be small if we can make $B$ small for positive values of $k_{x}$, while making it large for negative values of $k_{r}$. The function $B$ will

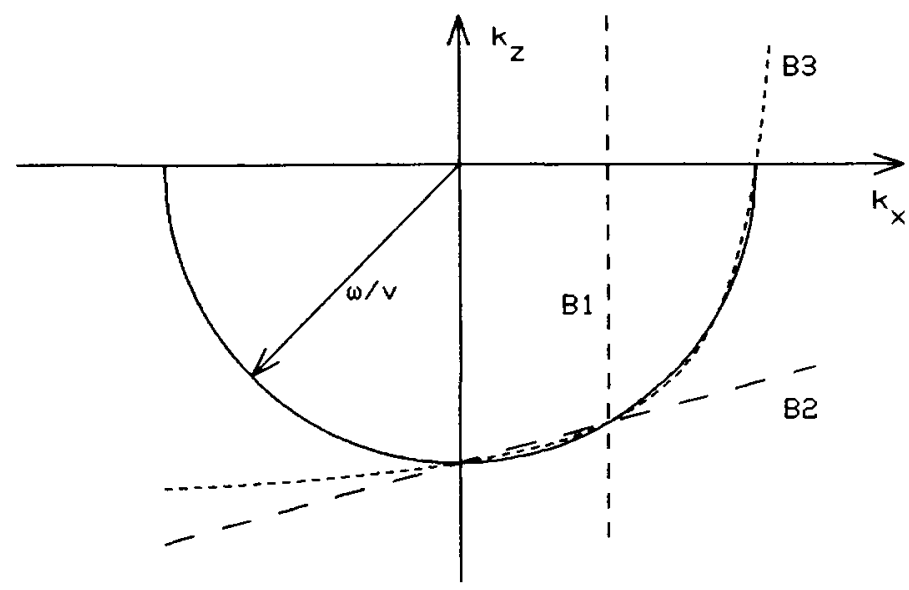

FIG. 2. Dispersion relations for absorbing boundary conditions. The curves labeled $B 1, B 2$, and $B 3$ are the dispersion relations for the unretarded absorbing boundary conditions presented in the text. The semicircle is from Figure 1, the exact paraxial dispersion relation. The dispersion curves are intended to be better approximations to the right quarter-circle than to the left quarter-circle. 


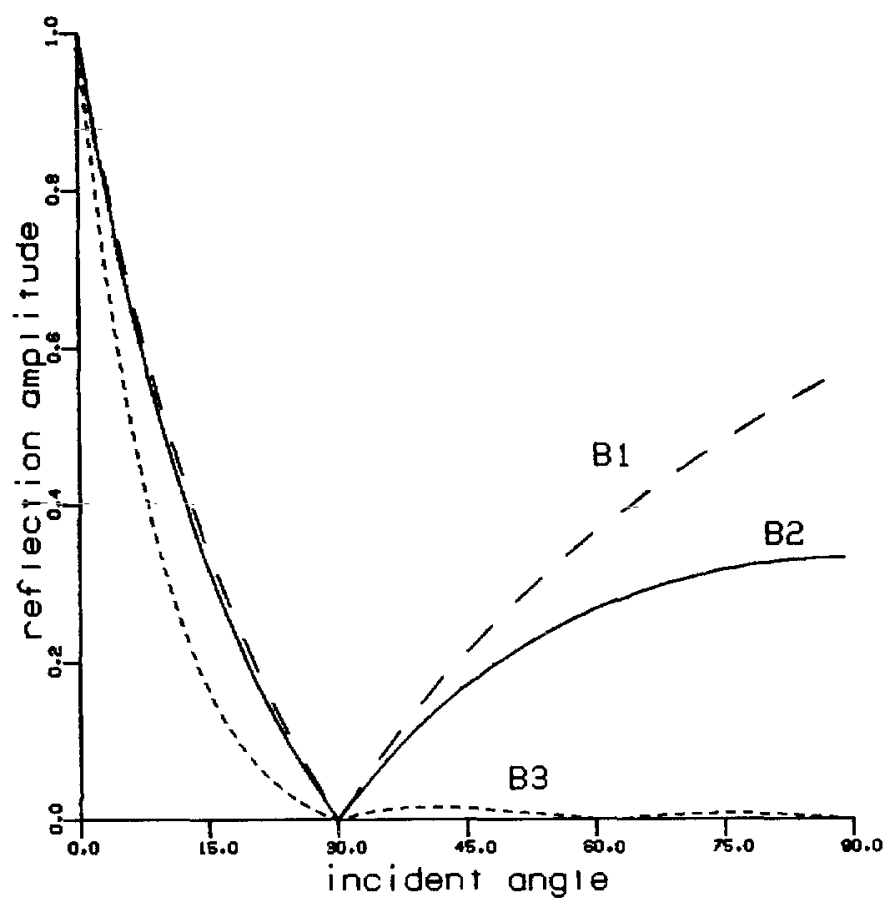

FIG. 3. Effective reflection coefficients for the absorbing boundary conditions. The effective reflection coefficients for the three types of absorbing boundaries, shown in Figure 2, are displayed as a function of incident angles at the boundary. The incident angle is defined such that flat events (perpendicular to sides on the section) have zero incident angle. The absorbing boundary conditions in this case were matched to the exact paraxial wave equation. The angles where the reflections vanish are fitting points of the boundary conditions.

be small when it closely matches the interior dispersion relation, that is, when $B\left(k_{,}, \omega\right)$ is small for a large range of $k_{x}, \omega>0$.

One constraint that we apply to $B$ is that it be no more than first order in $k_{r}$. In the physical domain, this implies that the boundary condition contains only first-order derivatives in $x$.

Three forms of boundary conditions meet the requirements for $B$ to be absorbing:

$$
\begin{aligned}
& B 1: \quad \frac{v k_{x}}{\omega}=b . \\
& B 2: \quad \frac{v k_{z}}{\omega}=-a+b \frac{v k_{x}}{\omega} . \\
& B 3: \quad \frac{v k_{z}}{\omega}=-\frac{a-b \frac{v k_{r}}{\omega}}{1-c \frac{v k_{x}}{\omega}} .
\end{aligned}
$$

The geometrical interpretation of $B 1$ is a vertical line in the $k_{r^{-}}, k_{z}$-plane, $B 2$ corresponds to a slanted line, and $B 3$ is one branch of a hyperbola. In Figure 2, the three boundary conditions are displayed along with the semicircle of the exact paraxial wave equation.

The coefficients $a, b$, and $c$ are determined by matching the boundary condition to the right side $\left(k_{, r}>0\right)$ of the dispersion relation of the interior equation. For monochromatic plane waves, the coefficients can be selected to annihilate perfectly any reflection. Since we wish to apply the boundary conditions to general wave fields, the coefficients are chosen to minimize ${ }_{\mid} R \mid$ for a range of wavenumbers which contains the band of significant energy in the wave tields. Although this range is problemdependent, the choice of coefficients is not critical.

The interior equation can be either the exact paraxial wave equation (3) or approximations thereof. The equations can be in a normal time frame [equations (6) and (7)], in retarded time [equations (9) and $(10)]$. or in the frequency domain. When re- 


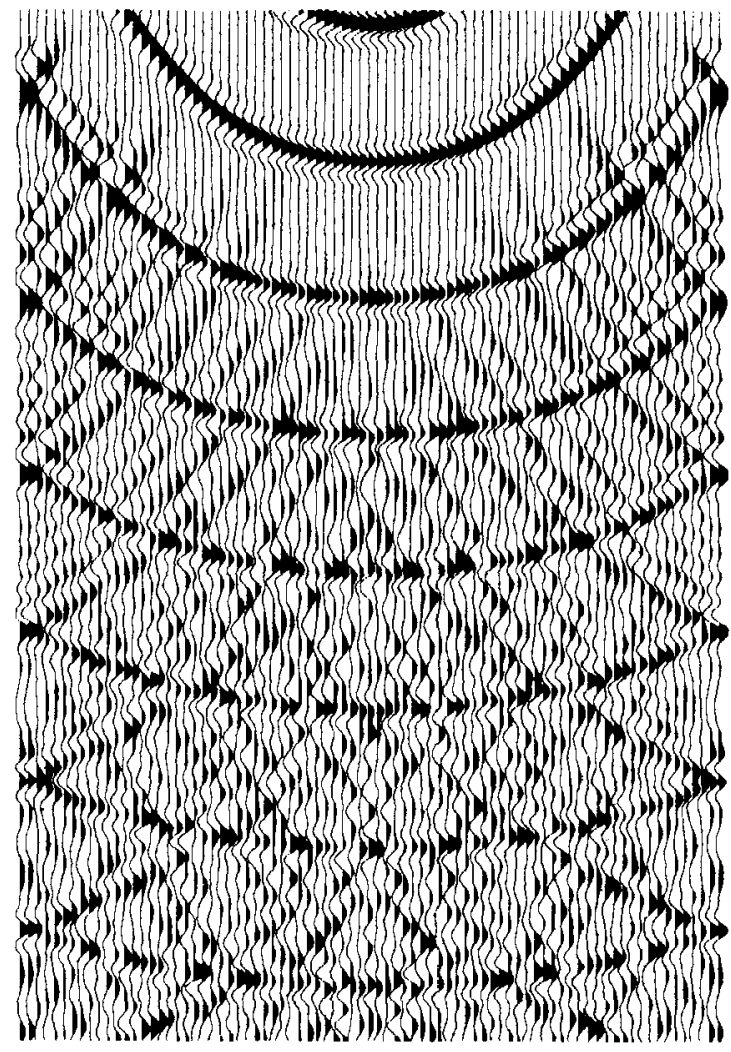

FIG. 4a. Monochromatic wave equation with reflecting boundary conditions. Shown is the downward continuation with the 45 -degree monochromatic wave equation of a point source location slightly above the top of the plot. The side boundaries are zero-slope Neumann conditions. The bottom portion of the plot is dominated by artificial reflections from the edges of the grid. Seven frequencies were added to enhance the clarity.

tarded coordinates are used, it is practical to fit the dispersion relation of the paraxial wave equation at $k_{r}=0\left[B\left(k_{r}=0, \omega\right)=0\right]$ since from this $a=0$, which simplifies the boundary conditions $B 2$ and B3.

In differential form, the three boundary conditions are:

$B 1: \quad P_{. r}+\frac{b}{\gamma} P_{t}=0$

B2: $\quad P_{z}-b P_{. x}-\frac{a}{v} P_{t}=0 ;$ and

B3: $\quad P_{z t}+c v P_{x z}-b P_{r t}-\frac{a}{v} P_{t t}=0$.

The effectiveness of the absorbing boundary conditions can be judged by examining the effective reflection coefficients as a function of incident angle to the boundary $\left[\sin ^{1}\left(1 k_{r} / \omega\right)\right]$. The method for calculating the reflection coefficients is given by equation (15). In Figure 3, these reflection coefficients are shown for the case when the interior equation is the exact paraxial equation. The angles for which there are no reflections are the fitting points for determining the coefficients in equations (19), (20), and (21). It should be noted that flat reflectors (zero angle of incidence) always have unit reflection coefficients. This can be seen from Equation (15). because the numerator and denominator are identical. For reflections with negative angles of incidence, the effective reflection coefficient is greater than unity. These reflections, however, do not cause stability problems because they migrate toward the interior of the mesh, rather than impinging on the boundary. A proof of stability is given in the Appendix. 


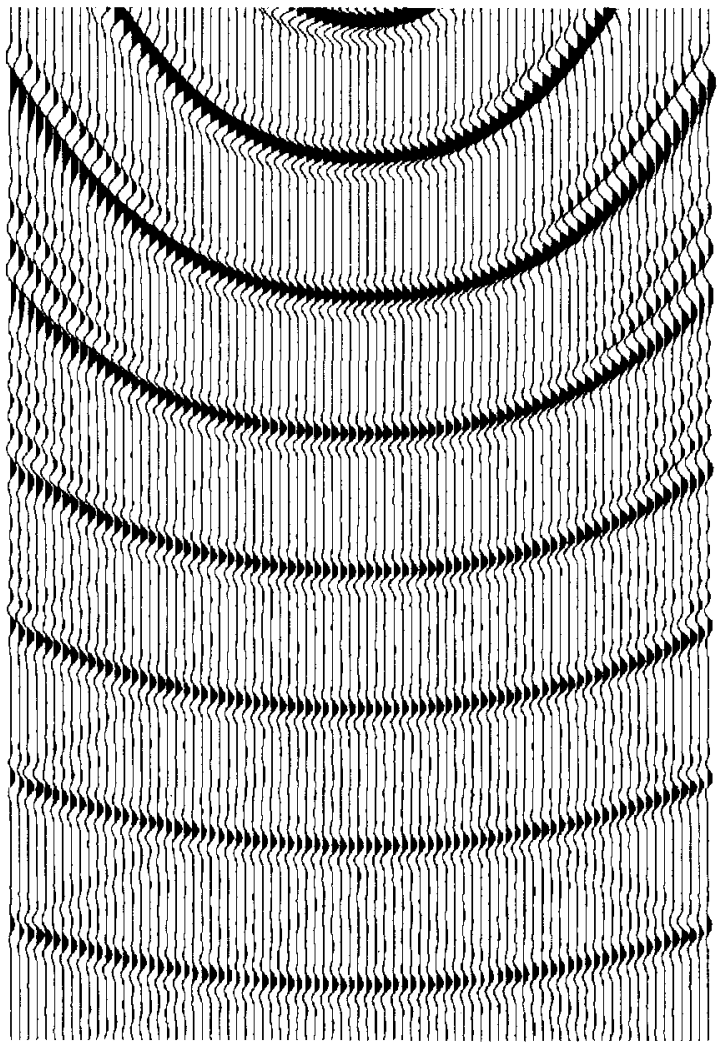

Fig. 4b. Monochromatic wave equation with $B 1$ absorbing boundary conditions. The same example as in Figure $4 \mathrm{a}$, except with $B 1$ absorbing boundary conditions. Only small artificial reflections are present.

The left side boundary is treated in a similar manner. The same formulas are valid if $b$ and $c$ are replaced with $-b$ and $-c$, respectively. For absorbing boundary conditions for downgoing waves, the $k_{z}$ in equations (17) and (18) is replaced by $-k_{z}$.

\section{NUMERICAL EXAMPLES}

To illustrate the effectiveness of the absorbing boundary conditions, two numerical examples are presented. The first example, shown in Figure 4, is a downward continuation using the monochromatic 45-degree wave equation. In the displays shown, solutions for seven different frequencies were combined to enhance the clarity of the plots. The point source located just above the top of each panel is used for the initial condition. Figure ta shows the results of downward continuing with zero-slope (perfectly reflecting) end conditions. The solution in the lower part of the mesh is dominated by reflections from the boundaries. In Figure $4 b$, the $B 1$ absorbing boundary condition was used, with the constant in the boundary condition adjusted to absorb perfectly at 60 degrees. This boundary condition removes most of the spurious reflections, especially those reflections at high angles of incidence. The shallow incidence angles do, however, generate some reflected energy. In the last panel, Figure $4 c$, the $B 3$ boundary condition was used. The fitting angles for the boundary condition were 24 and 60 degrees. The small reflections that remained with the $B 1$ boundary condition are now removed. The result of using the $B 2$ absorbing boundary condition is nearly identical to the $B 1$ result, and it is not shown.

The second example, shown in Figure 5, is a timedomain solution with the 15-degree paraxial wave equation. The first panel (A) shows the results of modeling the response of three point diffractors with the 15-degree wave equation. The $B 3$ absorbing 


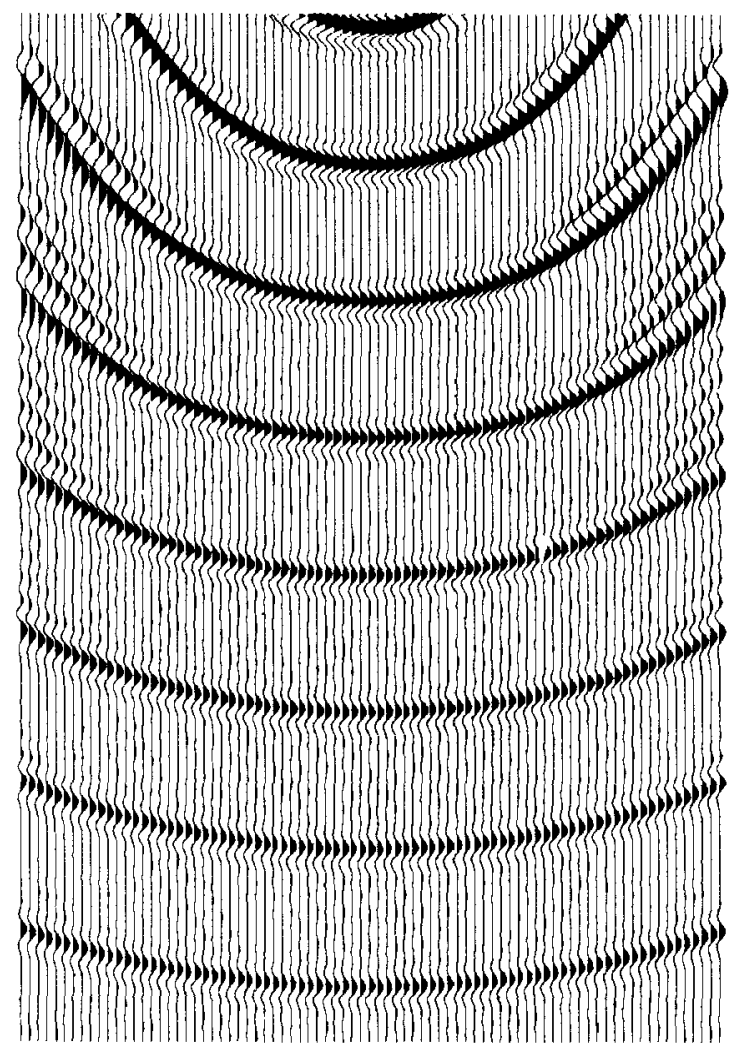

FIG. $4 c$. Monochromatic wave equation with $B 3$ absorbing boundary conditions. The example of Figure $4 \mathrm{a}$ is run with $B 3$ absorbing boundary conditions. Virtually all artificial reflections are removed.

boundary condition was used at the boundaries. The remaining panels $(B-E)$ are the results of migrating the data within the rectangular window. The correct result of migration should be that all energy focuses outside the window; some unfocused energy, however, will remain because of the energy lost on the sides in the modeling process.

In panel B, a zero-slope boundary condition was used, and clearly the artificial reflection dominated the result. The result obtained by padding with zero traces on the left is shown in panel $\mathrm{C}$. The boundary reflections are reduced significantly, but in this example it doubled the computational cost. The results of using the $B 1$ and $B 3$ absorbing boundary conditions are shown in panels $D$ and $E$, respectively. The boundary conditions remove most of the refections, with $B 3$ performing best.

\section{CONCLUSIONS}

Absorbing boundaries are most important in appli- cations where there are a limited number of traces in the section. Our experiments and analysis show that absorbing side boundary conditions can be applied to wave-equation migration with the following accomplishments:

(1) The nonphysical spurious reflections at the computational side boundaries are substantially reduced over the level of standard boundary conditions. With the most effective absorbing boundary condition $(B 3)$, the reflections are, for practical purposes, completely removed.

(2) The absorbing boundary conditions can be added in such a way that the full algorithm is computationally stable.

(3) The complexity of the algorithm is not increased by introducing absorbing boundaries. The boundary conditions are local (involving only a few neighboring mesh points) and have a 

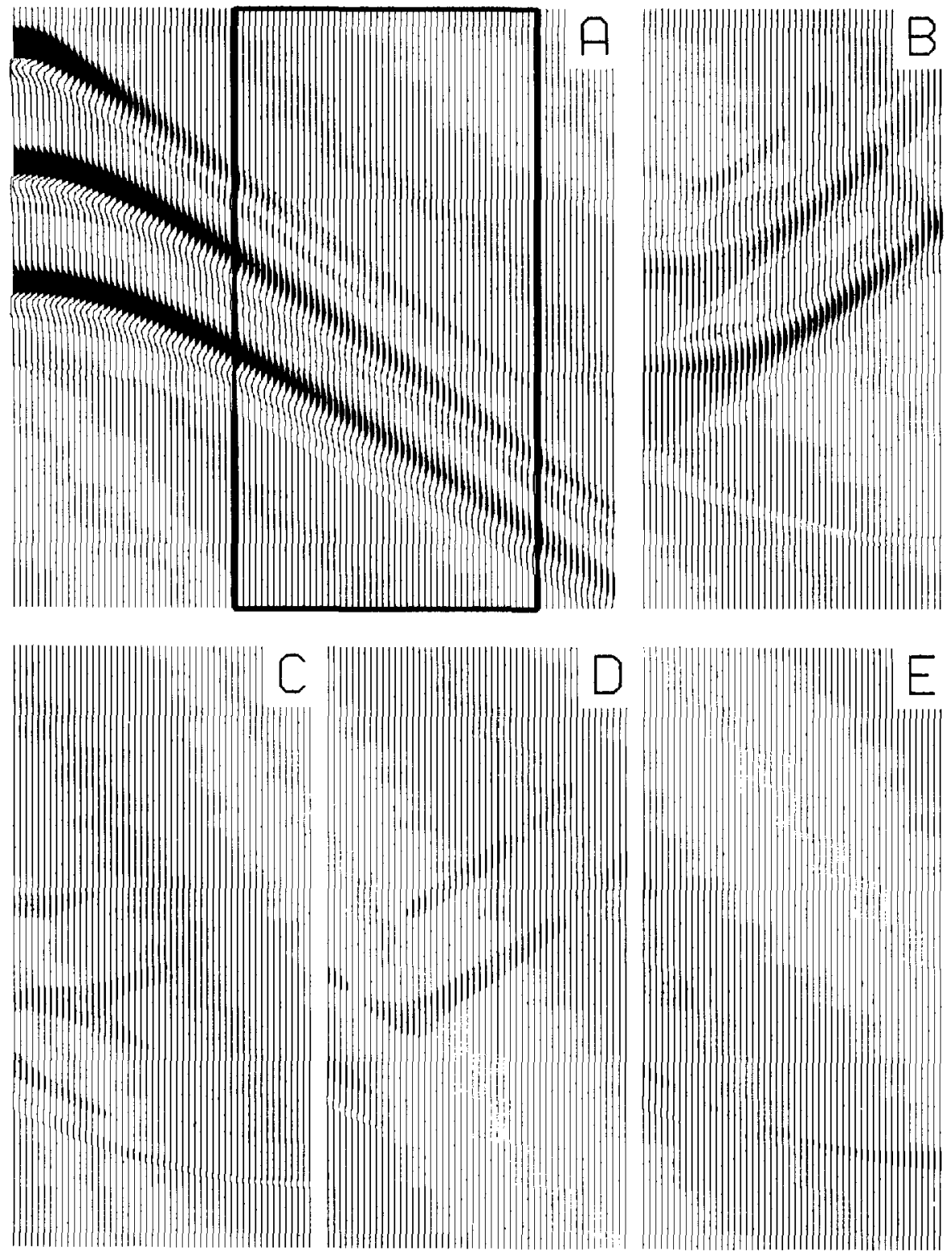

FIG. 5. A time-domain example of absorbing boundary conditions. In panel $\mathrm{A}$, the result using the 15 -degree time-domain wave equation to model the response of three point diffractors is shown. The $B 3$ absorbing boundary conditions were used at the edges to reduce spurious reflections. The remaining panels are the results of migrating data within the rectangular window with a 15 -degree wave equation. Plot scales for all panels are identical. The reflecting boundary case shown in panel B shows large artificial reflections from the left boundary. In panel $C$, the results of padding zero traces to the left side of the window are shown. In panels $\mathrm{D}$ and $\mathrm{E}$, the $B 1$ and $B 3$ absorbing boundaries, respectively, are used. 
finite-difference stencil similar to the scheme in the interior.

\section{ACKNOWLEDGMENTS}

Support for this research was provided by the sponsoring members of the Stanford Exploration Project. The authors also wish to thank Prof. Jon F. Claerbout for suggesting the $B 3$ boundary condition.

\section{REFERENCES}

Clacrbout. J. F., 1970, Coarse grid calculations of waves in inhomogeneous media with application to delineation of complicated seismic structure: Geophysics, v. 35, p. 407
1976. Fundamentals of geophysical data processing: New York, McGraw-Hill Book Co., Inc.

Claerbout. J. F., and Johnson, A. G., 1971, Extrapolation of time dependent waveforms along their path of propagation: Geophys. J. Roy. Astron. Soc.. v. 26, p. 285295.

Clayton, R. W., and Engquist, B., 1977. Absorbing boundary conditions for acoustic and elastic wave equations: SSA Bull., v. 6. p. 1529-1540.

Engquist, B., and Majda, A., 1977. Absorbing boundary conditions for the numerical simulation of waves: Math. Comp.. v. 31, p. 629-651.

Reynolds, A. C., 1978. Boundary conditions for the numerical solution of wave propagation problems: Geophysics, v. 43, p. 1099-1110.

Richtmyer, R. D., and Morton, K. W.. 1957, Difference methods for initial-value problems: New York. John Wiley and Sons, p. 135-1.37.

\section{APPENDIX \\ FORMULAS FOR BOUNDARY CONDITIONS AND STABILITY PROOF}

\section{Finite-difference formulas}

In order to compute the wave field $P(x, z, t)$, we discretize the differential equations and approximate $P(x, z, t)$ by a mesh function $P_{j, k}^{n}$ on the computational mesh $\left(x_{k}, z^{n}, t_{j}\right) ; x_{k}=A+k \Delta x, z^{n}=n \Delta z$, $t_{j}=j \Delta t ; k=0,1, \ldots, K ; n=0,1, \ldots ; j=\ldots$, $-1,0,1, \ldots$ The notation will be simplified if we introduce the usual forward $\left(D_{+}^{\xi}\right)$ and backward $\left(D_{-}^{\xi}\right)$ divided-difference operator with respect to the independent variable $\xi$. For example:

$$
D_{+}^{x} P_{j, k}^{n}=\left(P_{j, k+1}^{n}-P_{j, k}^{n}\right) / \Delta x,
$$

and

$$
D_{-}^{z} P_{j, k}^{n}=\left(P_{j, k}^{n}-P_{j, k}^{n-1}\right) / \Delta z
$$

Let us now define the difference approximations corresponding to the differential equations (9) and (10) which are used here.

\section{5-degree approximation, implicit}

$$
\begin{aligned}
D_{+}^{t} D_{+}^{z} P_{j, k}^{n}+ & \frac{v}{8} D_{+}^{x} D_{-}^{x}\left(P_{j, k}^{n+1}+\right. \\
& \left.P_{j+1, k}^{n+1}+P_{j, k}^{n}+P_{j+1, k}^{n}\right)=0 .
\end{aligned}
$$

\section{5-degree approximation, frequency domain}

$$
\begin{aligned}
& \omega^{2} D_{+}^{z} P_{k}^{n}(\omega)+\frac{v^{2}}{4} D_{+}^{x} D_{-}^{x} D_{+}^{z} P_{k}^{n}(\omega) \\
& +\frac{i \omega v}{4} D_{+}^{x} D_{-}^{x}\left[P_{k}^{n}(\omega)+P_{k}^{n+1}(\omega)\right]=0 .
\end{aligned}
$$

The difference equations $(\mathrm{A}-1)$ and $(\mathrm{A}-2)$ can be applied at all mesh points except for the side boundaries at $k=0$ and $k=K$. The difference formulas for the absorbing side boundaries at $k=K$ are (the formulas for $k=0$ are similar):

$$
\begin{aligned}
B 1: D_{-}^{x} & \left(P_{j, K}^{n}+P_{j+1, K}^{n}\right) \\
& +\frac{b}{\mathfrak{v}} D_{+}^{t}\left(P_{j, k}^{n}+P_{j, K-1}^{n}\right)=0 ; \quad(\mathrm{A}-3)
\end{aligned}
$$

B2: $\quad D_{+}^{z}\left(P_{j, K}^{n}+P_{j+1, K}^{n}+P_{j, K-1}^{n}+P_{j+1, K-1}^{n}\right)$

$$
-\frac{a}{v} D_{+}^{t}\left(P_{j, K}^{n}+P_{j, K-1}^{n}+P_{j, K}^{n+1}\right.
$$$$
\left.+P_{j, K-1}^{n+1}\right)-b D_{-}^{x}\left(P_{j, K}^{n}+P_{j+1, K}^{n}\right.
$$$$
\left.+P_{j, K}^{n+1}+P_{j+1, K}^{n+1}\right)=0 ;
$$

$$
\begin{aligned}
B 3: & (a=0) \\
D_{+}^{z} & D_{+}^{t}\left(P_{j, K}^{n}+P_{j, K-1}^{n}\right) \\
+ & c v D_{-}^{x} D_{+}^{z}\left(P_{j, K}^{n}+P_{j+1, K}^{n}\right) \\
& -b D_{-}^{r} D_{+}^{t}\left(P_{j, K}^{n}+P_{j, K}^{n+1}\right)=0 .
\end{aligned}
$$

If more accurate difference formulas involving more mesh points are needed to approximate the $x$ derivative in the interior equation, the boundary conditions must be used also at the mesh points close to $x_{0}$ and $x_{K}$.

\section{Stability proof}

In order to be at all useful for computations, the difference scheme together with the boundary con- 
ditions must be stable. The computed solution should not be permitted to grow in an uncontrolled manner. We rigorously show that a norm of $P$ stays bounded for the interior scheme of equation $(A-1)$ together with the boundary condition $(A-3)$. We present the proof since similar analyses apply to other schemes and can be useful in the study of stable boundary conditions in general.

We define the $l_{2}$ norm of $P_{j, k}^{n}$ :

$$
\left\|P^{n}\right\|=\left[\Delta t \Delta x \sum_{j=-\infty}^{\infty} \sum_{k=1}^{K-1}\left(P_{j, k}^{n}\right)^{2}\right]^{1 / 2} .
$$

Using Parseval's relation, we realize that it is sufficient to bound the norm in the frequency domain

$$
\left\|P^{n}(\omega)\right\|=\left[\Delta x \sum_{k=1}^{K-1} P_{k}^{n}(\omega) \overline{P_{k}^{n}(\omega)}\right]^{1 / 2} .
$$

Assuming there is no dc component (bias) in $P$, we can rewrite the difference schemes in the frequency domain. For the interior equation, left absorbing boundary, and right absorbing boundary, we have, respectively

$$
\begin{aligned}
D_{+}^{z} P_{k}^{n} & =i \alpha D_{+}^{x} D_{-}^{x}\left(P_{k}^{n+1}+P_{k}^{n}\right)(\mathrm{A}-6) \\
-\frac{8 \alpha i}{b} D_{+}^{x} P_{0}^{n+1} & =\left(P_{0}^{n+1}+P_{1}^{n+1}\right) \\
\frac{8 \alpha i}{b} D_{-}^{x} P_{K}^{n+1} & =\left(P_{K}^{n+1}+P_{K-1}^{n+1}\right),
\end{aligned}
$$

where we have defined $\alpha=-v / 8 \Delta t \cot (\omega \Delta t / 2)$.

We now prove that the norm of $P$ will not increase from one $z$-step to the next. That is, the quantity

$$
\begin{gathered}
E=P^{n+1}\left\|^{2}-\right\| P^{n} \|^{2}=\Delta x \operatorname{Re} \sum_{k=1}^{K-1} . \\
\cdot\left(P_{k}^{n+1}+P_{k}^{n}\right)\left(\overline{P_{k}^{n+1}-P_{k}^{n}}\right)
\end{gathered}
$$

is less than or equal to zero. Using equation $(A-6)$, we have

$$
\begin{aligned}
E= & \Delta x \operatorname{Re} \sum_{k=1}^{K-1}\left(P_{k}^{n+1}+P_{k}^{n}\right) . \\
& . \overline{i \alpha \Delta z D_{+}^{x} D^{x}\left(P_{k}^{n+1}+P_{k}^{n}\right)} .
\end{aligned}
$$

We can now apply the summation by parts formula (Richtmyer and Morton, 1957) and drop the imaginary terms

$$
\begin{aligned}
E= & \frac{-\Delta z}{2} \alpha \operatorname{Re}\left\{i \left[\left(P_{K}^{n+1}+P_{K}^{n}\right.\right.\right. \\
& \left.\left.+P_{K-1}^{n+1}+P_{K-1}^{n}\right) D_{-}^{x}\left(\overline{P_{K}^{n+1}+P_{K}^{n}}\right)\right] \\
& -\left[\left(P_{1}^{n+1}+P_{1}^{n}+P_{0}^{n+1}\right.\right. \\
& \left.\left.\left.+P_{0}^{n}\right) D_{+}^{x}\left(\overline{P_{0}^{n+1}+P_{0}^{n}}\right)\right]\right\} .
\end{aligned}
$$

The boundary equations $(A-7)$ and $(A-8)$ are now substituted for the divided differences

$$
\begin{aligned}
E= & \frac{\Delta z b}{16}\left(\left|P_{K}^{n+1}+P_{K}^{n}+P_{K-1}^{n+1}+P_{K-1}^{n}\right|\right. \\
& \left.+\left|P_{1}^{n+1}+P_{1}^{n}+P_{0}^{n+1}+P_{0}^{n}\right|\right) \leq 0 .
\end{aligned}
$$

Hence, the norm does not increase with $z$, and the scheme is stable for $b \leq 0$, which is the right sign for absorbing boundary conditions in computations where $n$ increases. 